\title{
Cellular target recognition of perfluoroalkyl acids: In vitro evaluation of inhibitory effects on lysine decarboxylase
}

\author{
Sufang Wang, Qiyan Lv, Yu Yang *, Liang-Hong Guo *, Bin Wan, Lixia Zhao
}

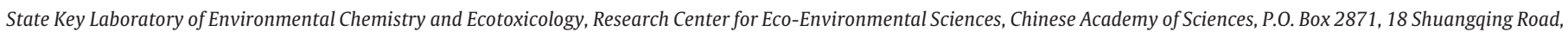
Beijing 100085, China

\section{H I G H L I G H T S}

- Inhibitory effects of PFAAs on lysine decarboxylase activity were evaluated.

- Four different methods were employed to investigate the mechanisms.

- The long chain PFAAs showed inhibitory effect compare with 4-6 carbon chain.

- The long chain PFAAs bound with LDC differently from the short ones.

- The results in cells correlate with those obtained from fluorescence assay.

\section{G R A P H I C A L A B S T R A C T}
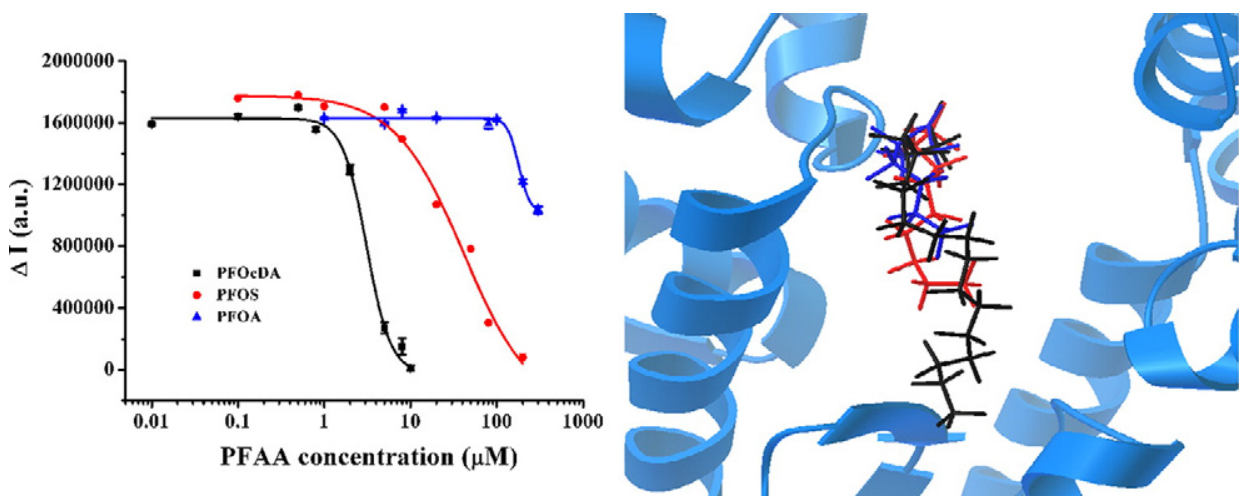

\begin{abstract}
A B S T R A C T
Perfluoroalkyl acids (PFAAs) have been shown to bind with hepatic peroxisome proliferator receptor $\alpha$, estrogen receptors and human serum albumin and subsequently cause some toxic effects. Lysine decarboxylase (LDC) plays an important role in cell growth and developmental processes. In this study, the inhibitory effect of 16 PFAAs, including 13 perfluorinated carboxylic acids (PFCAs) and 3 perfluorinated sulfonic acids (PFSAs), on lysine decarboxylase (LDC) activity was investigated. The inhibition constants obtained in fluorescence enzyme assays fall in the range of $2.960 \mu \mathrm{M}$ to $290.8 \mu \mathrm{M}$ for targeted PFCAs, and $41.22 \mu \mathrm{M}$ to $67.44 \mu \mathrm{M}$ for targeted PFSAs. The inhibitory effect of PFCAs increased significantly with carbon chain (7-18 carbons), whereas the short chain PFCAs (less than 7 carbons) did not show any effect. Circular dichroism results showed that PFAA binding induced significant protein secondary structural changes. Molecular docking revealed that the inhibitory effect could be rationalized well by the cleft binding mode as well as the size, substituent group and hydrophobic characteristics of the PFAAs. At non-cytotoxic concentrations, three selected PFAAs inhibited LDC activity in HepG2 cells, and subsequently resulted in the decreased cadaverine level in the exposed cells, suggesting that LDC may be a possible target of PFAAs for their in vivo toxic effects.
\end{abstract}

(C) 2014 Elsevier B.V. All rights reserved.

\footnotetext{
* Corresponding authors. Tel./fax: + 861062849685.

E-mail addresses: yuyang@rcees.ac.cn (Y. Yang), LHGuo@rcees.ac.cn (L.-H. Guo).
}

\section{Introduction}

Perfluoroalkyl acids (PFAAs) are a family of fluorine-containing chemicals with unique physical and chemical properties that have been widely used in industrial and commercial products. Two most widely known PFAAs are perfluorooctane sulfonate (PFOS) and 
perfluorooctanoic acid (PFOA). Due to the extraordinary stability of the carbon-fluorine bond, these compounds are resistant to chemical, biological and thermal degradation. Therefore, PFAAs have been found in environments and accumulate in the bodies of humans and wildlife (Giesy and Kannan, 2002; Sundström et al., 2011; Raymer et al., 2012; Houde et al., 2006). A number of studies have demonstrated the adverse effects of PFAAs on experimental animals, mostly of PFOA and PFOS (Lau et al., 2007). In 2009, PFOS was added to the list of persistent organic pollutants of the Stockholm Convention to reduce and eventually eliminate its production and use. In spite of all these policies and bans, due to the nature of persistence, bioaccumulation and biomagnifications through the food chain (Conder et al., 2008) of PFAAs, their risks to human health are still worthy of long-term concern.

Numerous studies have been published regarding the toxicity and human health effects of PFAAs, including loss of body weight, hepatotoxicity, interference of lipid transportation and metabolism, endocrine effects, tumorigenicity, reproductive toxicity and developmental toxicity (Thorsten et al., 2011). Early toxicological studies of PFAAs (fatty acids like structures) focused on the mechanisms involving liganddependent activation of the hepatic peroxisome proliferator receptor $\alpha$ (PPAR $\alpha)$, which induces enzymes responsible for $\beta$-oxidation, fatty acid $\omega$-oxidation and cholesterol homeostasis (Andersen et al., 2008; DeWitt et al., 2009). However, due to the observation of increased liver cancer incidence by PFAAs in PPAR $\alpha$ knockout mice, the role of estrogenic activity of PFAAs in their hepatocarcinogenesis has attracted increasing attentions recently (Ito et al., 2007; Guyton et al., 2009). Some PFAAs were found to bind to estrogen receptors (ERs) and recruit coactivator peptides in vitro, and induce ER-mediated transcriptions in cells (Benninghoff et al., 2011; Gao et al., 2013). Several studies also found depression of thyroid hormone levels in PFOS-exposed rats (Lau et al., 2003; Wang et al., 2011). The interactions between PFAAs and thyroid hormone transport proteins were also observed (Chang et al., 2007). In vitro binding between PFAAs and human serum albumin was also evaluated as a possible route for disrupting fatty acid transport in blood (Chen and Guo, 2009; Hebert and MacManus-Spencer, 2010). Although some biological targets have been gradually revealed for exploring molecular mechanisms underlying PFAA toxicity, there are still other proteins in vivo to potentially interact with PFAAs (Weiss et al., 2009; White et al., 2011; Zhang et al., 2013).

Lysine decarboxylase (LDC) is a key enzyme involved in the production of cadaverine by the decarboxylation of lysine. Cadaverine is known to be involved in a number of growth and developmental processes (Bagni and Tassoni, 2001) and the biosynthesis of a broad range of alkaloids (Leistner and Spenser, 1973) with beneficial pharmacological properties, such as cytotoxic, antiarrhythmic, oxytocic, hypoglycemic, and antipyretic activities (Ohmiya et al., 1995; Michael, 2008). Inhibition of cadaverine synthesis suppressed wound healing in rodents (Calandra et al., 1996). Some diseases including cancers are closely associated with the abnormal cadaverine level (Gerner and Meyskens, 2004). Although LDC plays an important role in many biological processes, information on the molecular regulatory mechanisms is limited.

In the present study, we employed a fluorescence sensing method to measure the inhibitory effect of 16 PFAAs on LDC activity. The selected PFAAs are structurally diverse, with different carbon chain lengths (418 carbons) and functional groups (carboxylic or sulfonic acid). By combining the fluorescence sensing assay, circular dichroism (CD) spectroscopy, in vitro cell experiments and molecular docking, the relationship between PFAA structure and their LDC inhibitory effect, as well as LDC as a potential cellular target of PFAAs, was investigated and analyzed.

\section{Material and methods}

\subsection{Chemicals}

Lysine, cadaverine, cucurbit[7] uril (CB7), lysine decarboxylase (LDC, from Bacterium cadaveris), 2,4,6-trinitrobenzenesulfonic acid (TNBS), 1,7-diaminoheptane (DAH), 3-(4, 5-dimethyl-2-thiazolyl)-2, 5diphenyl-2H-tetrazolium bromide (MTT), perfluorobutyric acid (PFBA, 98\%), perfluoropentanoic acid (PFPA, 97\%), perfluorohexanoic acid (PFHxA, $\geq 97 \%$ ), perfluoroheptanoic acid (PFHpA, 99\%), perfluorooctanoic acid (PFOA, 96\%), perfluorononanoic acid (PFNA, 97\%), perfluorodecanoic acid (PFDA, 98\%), perfluoroundecanoic acid (PFUnA, 95\%), perfluorododecanoic acid (PFDoA, 96\%), perfluorotridecanoic acid (PFTrDA, 97\%), perfluorotetradecanoic acid (PFTeDA, 97\%), perfluorobutane sulfonate (PFBS, 97\%), perfluorohexane sulfonate (PFHxS, $\geq 98.0 \%$ ) and perfluorooctane sulfonate (PFOS, $\geq 98.0 \%$ ) were purchased from Sigma-Aldrich (St. Louis, MO, USA). Perfluorohexadecanoic acid (PFHxDA, 95\%) and perfluorooctadecanoic acid (PFOcDA, 97\%) were obtained from Alfa Aesar (Ward Hill, MA, USA). Dapoxyl was from Molecular Probes (Eugene, OR, USA). Guanosine 5'-diphosphate, 3'-diphosphate (ppGpp) was used as received from Trilink BioTechnologies (San Diego, CA). (Fig. S1 in the supplementary data) Benzoyl chloride was from TCI (Shanghai, China). All chemicals are of analytical grade. BCA protein assay kit was obtained from ComWin Biotech (Beijing, China).

\subsection{Fluorescence enzyme activity and inhibition assay}

To investigate the inhibitory effect of PFAAs on LDC, a proper enzyme activity assay is needed. In 2007, Henning et al. described a novel concept for the determination of LDC enzymatic activity, using macrocyclic receptors and fluorescent dyes such as CB7/Dapoxyl and CX4/DBO (Henning et al., 2007). This product-competitive displacement method is simple, convenient and label-free. Briefly, a selfassembled host-guest inclusion complex CB7/Dapoxyl was employed as a fluorescence reporter pair for the enzymatic activity assay of LDC. Competitive binding of the enzyme product cadaverine with CB7 displaces Dapoxyl from CB7, leading to reduced fluorescence intensity. If the enzymatic activity is inhibited, the fluorescence intensity would remain unchanged (Scheme 1). LDC activity assay was performed according to the following protocol. The reaction mixture containing $20 \mu \mathrm{gL}^{-1} \mathrm{LDC}, 50 \mu \mathrm{M}$ lysine, $2.5 \mu \mathrm{M}$ Dapoxyl and $30 \mu \mathrm{M}$ CB7 in a total volume of $100 \mu \mathrm{L}$ was incubated at $37^{\circ} \mathrm{C}$ for $1.5 \mathrm{~h}$. For the inhibition assay, inhibitors at varying concentrations were included in the reaction mixture. The change in fluorescence intensity $\left(\Delta \mathrm{I}=\mathrm{I}_{0}-\mathrm{I}\right)$ was taken as relative LDC activity, and was plotted as a function of inhibitor concentration. $\mathrm{I}$ and $\mathrm{I}_{0}$ are the fluorescence intensity of CB7/Dapoxyl in the presence and absence of LDC. The dose-response curve was fitted with a sigmoidal model (Origin Lab 8.0, Northampton, MA, USA) and analyzed with the Hill equation to obtain $\mathrm{IC}_{50}$ value (Copeland, 2003), which can be readily converted into the inhibition constants $K_{\mathrm{i}}$ by considering the enzyme concentration (Nau et al., 2009):

$I C_{50}=K_{i}+\frac{1}{2}[E]$

Steady-state fluorescence was measured on a Horiba FluoroMax-4 spectrofluorimeter (Edison, NJ, USA). The excitation and emission wavelength were $336 \mathrm{~nm}$ and $380 \mathrm{~nm}$, respectively. Excitation and emission slits were both set at $4 \mathrm{~nm}$.

\subsection{Circular dichroism spectroscopic measurement}

Circular dichroism (CD) spectra measurement of LDC $\left(150 \mu \mathrm{gL}^{-1}\right.$ in $\mathrm{HCl}-\mathrm{NH}_{4} \mathrm{OAc}$ buffer) was carried out in the absence and presence of PFAAs on a JASCO J-815 spectropolarimeter (Tokyo, Japan) with a $10 \mathrm{~mm}$ path length quartz cuvette. The $\mathrm{CD}$ spectra were recorded from 190 to $300 \mathrm{~nm}$ at a scan rate of $50 \mathrm{~nm} / \mathrm{min}$ and $1 \mathrm{~s}$ response time. Three scans were averaged for protein secondary structure analysis, which was performed with the JWSSE- 513 program installed on the $\mathrm{CD}$ instrument. In the experiments, different concentrations of PFAAs in acetonitrile were incubated with LDC in $\mathrm{NH}_{4} \mathrm{OAc}$ buffer. The final 


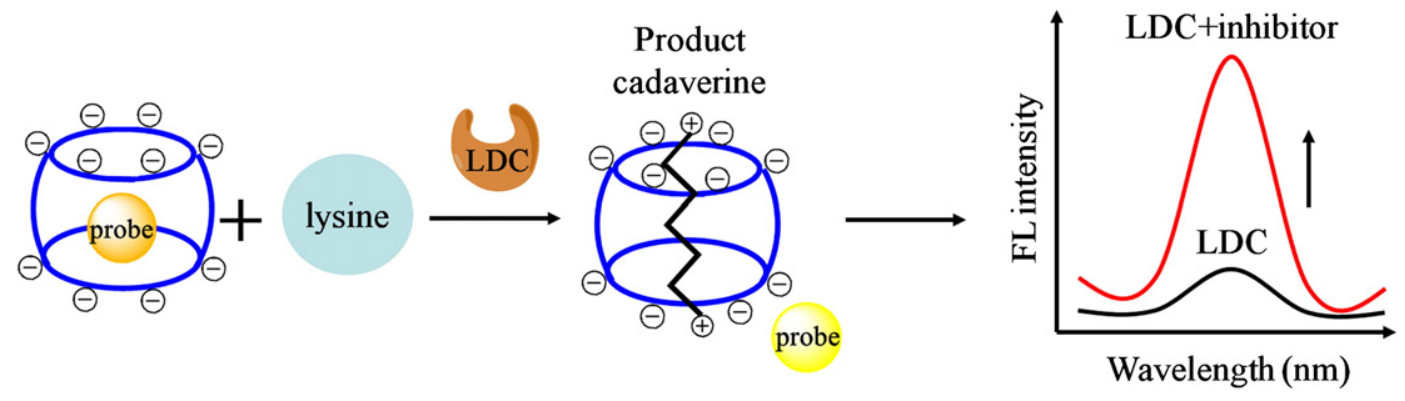

Scheme 1. Scheme describing the design of label-free fluorescence sensing mechanism for the assay of inhibitory effect of PFAAs on LDC activity.

content of the organic solvent was kept below $2 \%$ to minimize potential interference.

\subsection{Cell culture, treatment and viability assay}

Human cell line HepG2 was selected based on the hepatotoxicity of PFAAs. The cells were purchased from ATCC (VA, USA) and maintained in complete RPMI-1640 (cRPMI-1640) containing 10\% heat-deactivated fetal bovine serum (GIBCO, Invitrogen Life Technology, NY, USA), penicillin and streptomycin (100 $\mathrm{U} \mathrm{mL}^{-1}$ each). Cells within 20 passages were used for experiments and cultured at $37{ }^{\circ} \mathrm{C}$ in a humidified atmosphere of $95 \%$ air and $5 \% \mathrm{CO}_{2}$. Cells were seeded at approximated $0.5-1 \times 10^{5}$ cells $\mathrm{mL}^{-1}$ in 96 -well plates for cell viability assay. The exposure was performed in cRPMI with or without (DMSO control) PFOS, PFOA, PFBA (up to $500 \mu \mathrm{M}$ ), and $25 \mu \mathrm{M}$ for PFOcDA, respectively. The exposure lasted for $24 \mathrm{~h}$ unless specified elsewhere.

The viability change of the cells under $24 \mathrm{~h}$ exposure of PFAAs at various concentrations was determined by MTT. In brief, after the exposure, MTT reagents (1:10 dilution) were added into each well of the 96-plate, followed by $4 \mathrm{~h}$ incubation at $37^{\circ} \mathrm{C}$. The absorbance data were then recorded on a Varioskan Flash microplate reader (Thermo Fisher Scientific, Waltham, MA, USA) at $570 \mathrm{~nm}$.

\subsection{LDC activity assay in cells}

Since the catalytic residues are highly conserved among species, therefore, the effects of PFAAs on standard LDC catalytic activity might reflect the influence of these chemicals on human cells. To confirm the effect of PFAAs on LDC activity in human cells, we determined the LDC enzymatic activity after PFAA exposure. We assessed the LDC activity in HepG2 cells by a spectrophotometric assay described previously (Phan et al., 1982). It is based upon the principle that cadaverine, a product of lysine decarboxylase (Phan et al., 1982; Kanjee et al., 2011), reacts with TNBS to give a product soluble in toluene whereas the enzyme substrate lysine does not. Cells were treated with various concentrations of PFOcDA (0-25 $\mu \mathrm{M})$, PFOS (0-100 $\mu \mathrm{M})$, PFOA (0-200 $\mu \mathrm{M})$, PFBA (0$200 \mu \mathrm{M}$ ) or control medium (VC) for $24 \mathrm{~h}$. After PFAA exposure, the cells were lysed in a buffer containing $0.125 \mathrm{M}$ Tris- $\mathrm{HCl}$ ( $\mathrm{pH} \mathrm{6.8),} \mathrm{4 \%}$ SDS, $20 \%$ glycerol, $2 \%$ 2-mercaptoethanol, and centrifuged to obtain the supernatant. The protein content in cell lysate was then quantified by BCA protein assay kit. Appropriate amounts of cell extracts were mixed with lysine and then incubated for $1 \mathrm{~h}$ at $37^{\circ} \mathrm{C}$ in $10 \mathrm{mM}$ potassium phosphate buffer ( $\mathrm{pH}$ 6.0). To each sample, $\mathrm{K}_{2} \mathrm{CO}_{3}$ was added to terminate the reaction, and TNBS was added to produce an intensely colored product, $\mathrm{N}, \mathrm{N}^{\prime}$-bistrinitrophenylcadaverine (TNP-cadaverine), and the samples were incubated for $5 \mathrm{~min}$ at $40{ }^{\circ} \mathrm{C}$. Each sample was thoroughly mixed with toluene and separated. The toluene layer was used for subsequent UV-vis analysis. The control level is the signal of PBS solution containing cell lysate, $\mathrm{K}_{2} \mathrm{CO}_{3}$ and TNBS in the absence of lysine. UV-visible absorption spectra were measured on an Agilent 8453 UV-vis spectrophotometer (Santa Clara, CA, USA).

\subsection{Change of cadaverine level in exposed cells}

The amount of cadaverine in control and exposed cells was measured by high-performance liquid chromatography (HPLC) (Agilent 1260, Hewlett Packard, Wilmington, NC, USA) as described in earlier papers (Flores and Galston, 1982; Liu et al., 2010). Cells were solubilized in lysis buffer after $24 \mathrm{~h}$ exposure. The cell suspension was then centrifuged at $8000 \mathrm{~g}$ for $20 \mathrm{~min}$ and the supernatant was derivatized with benzoyl chloride, using DAH as the internal standard. Samples were injected into an Eclipse Plus C18 column (50 $\mathrm{mm} \times 3 \mathrm{~mm}$; 20/80 water/acetonitrile; $0.1 \mathrm{~mL} / \mathrm{min}, 25^{\circ} \mathrm{C}$ ) with UV detector $(254 \mathrm{~nm})$ to determine the cadaverine concentration.

\subsection{Molecular docking}

The 3D crystal structure of LDC was obtained from the Protein Data Bank for constructing the receptor model (Kanjee et al., 2011). Sixteen PFAAs, as well as a known LDC inhibitor ppGpp, were docked to LDC using Lamarckian genetic algorithm provided by AutoDock 4.2 software (Morris et al., 1998, 2009). Grid boxes were centered at the interface between neighboring monomers ( $A$ and $C$ ) in the decamer of LDC and built with $60 \times 60 \times 60$ point cube coverage. A spacing of $0.375 \AA$ between the grid points was used. All other docking parameters were set to defaults, including a population size of 150 , maximum number of 2.5 million evaluations, maximum of 2700 generations, gene mutation rate of 0.02 , crossover rate of 0.8 and $10 \mathrm{GA}$ runs. For each compound, ten independent docking runs were carried out, and the one with the lowest binding energy was selected for analysis.

\subsection{Statistical analysis}

All the experiments were repeated for three times, and the data were expressed by mean \pm S.D. $n=3$. In the cell experiment, we analyzed $p$ values of the experimental data by a two-tailed paired T-test using the Microsoft Excel software. A $p$ value of less than 0.05 was considered statistically significant.

\section{Results and discussion}

\subsection{LDC activity by fluorescence sensing assay and validation}

We tested the possibility of using reporter pair CB7/Dapoxyl to determine the inhibitory effect of PFAAs on LDC activity. Based on the Dapoxyl fluorescence titration curve (Fig. S2 in the Supplementary data), the concentration of the CB7/Dapoxyl reporter pair in the following fluorescence displacement experiments was chosen to be $30 \mu \mathrm{M} / 2.5 \mu \mathrm{M}$. After the addition of cadaverine, the fluorescence intensity decreased significantly. However, no substantial change in the spectra was observed when lysine was added (Fig. S3 in the Supplementary data). The concentrations of LDC and substrate lysine were then optimized and the optimal concentrations of LDC and lysine were determined to be $20 \mu \mathrm{gL}^{-1}$ and 

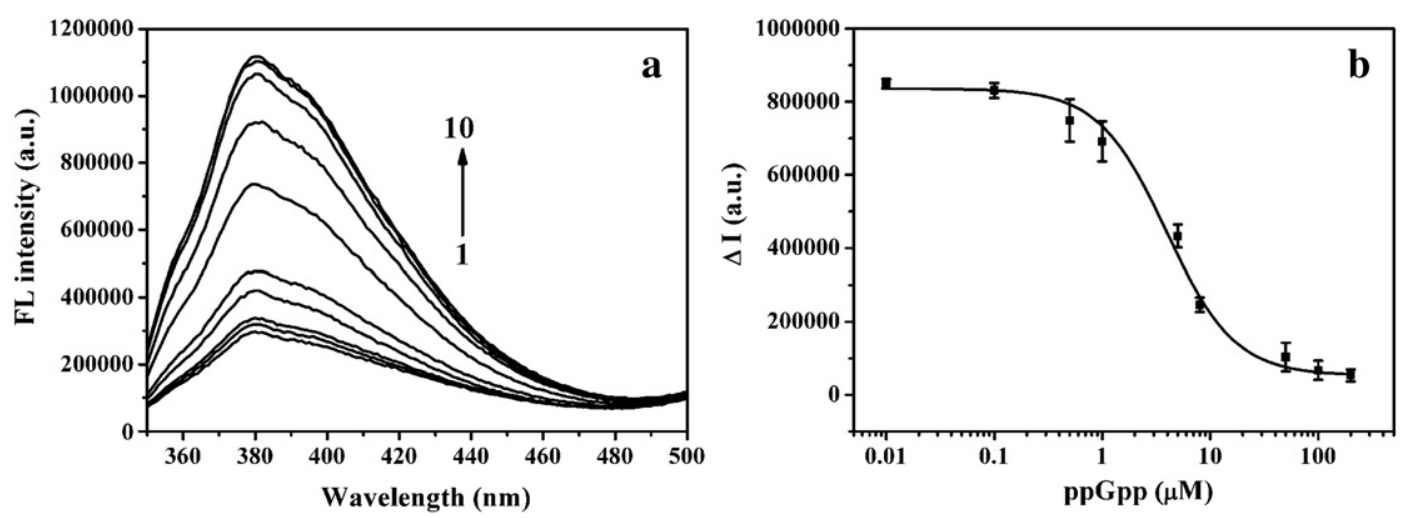

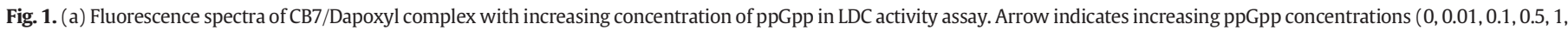

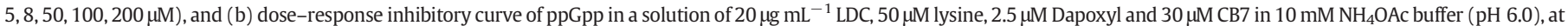
$37^{\circ} \mathrm{C}$. Data points represent the mean \pm S.D. $n=3$.

$50 \mu \mathrm{M}$ (Fig. S4 in the Supplementary data). In order to validate the method, the LDC activity in the presence of ppGpp, a natural inhibitor of LDC produced in vivo was investigated. As LDC and its inhibitor ppGpp were added into the mixed solution of $50 \mu \mathrm{M}$ lysine, $2.5 \mu \mathrm{M}$ Dapoxyl and $30 \mu \mathrm{M}$ CB7, Dapoxyl peak fluorescence intensity at $380 \mathrm{~nm}$ gradually increased until it reached a plateau (Fig. $1 \mathrm{a}$ ). The $\mathrm{IC}_{50}$ value (the concentration of ppGpp required to reduce LDC activity by 50\%) was found to be $3.940 \mu \mathrm{M}$ (Fig. 1b). Using the $\mathrm{IC}_{50}$ value, the inhibition constant $\left(K_{\mathrm{i}}\right)$ was calculated to be $3.810 \mu \mathrm{M}$ for $\mathrm{ppGpp}$. The result is close to that $\left(K_{\mathrm{i}}=0.68 \mu \mathrm{M}\right)$ reported in the literature (Kanjee et al., 2011), and thus verifies the effectiveness of this method.

\subsection{Inhibitory effect of PFAAs on LDC activity}

After the product-competitive fluorescence displacement method was validated, the inhibitory effect of 16 PFAAs on LDC activity was investigated. Inhibitory effect on the LDC activity was observed in a dose-dependent manner. According to the dose-effect relationship (Fig. S5 in the Supplementary data), the corresponding inhibition constants $K_{\mathrm{i}}$ were estimated. The results are listed in Table 1 . The inhibition constants fall in the range of 2.960 to $290.8 \mu \mathrm{M}$ for PFCAs and 41.22 to $67.44 \mu \mathrm{M}$ for PFSAs. Clearly, the inhibitory effect increased significantly with carbon number for medium (7-10 carbons) and long chain (11-18 carbons) PFCAs, whereas short chain PFCAs (less than 7 carbons) did not show any effect. The relationship between the inhibition constant

Table 1

Inhibition constant $K_{\mathrm{i}}$, octanol/water partition coefficient $\log K_{\mathrm{ow}}$ and molecular length of PFAAs.

\begin{tabular}{lllcc}
\hline PFAAs & Molecular formulae & $K_{\mathrm{i}}(\mu \mathrm{M})$ & ${\text { Log } K_{\text {ow }}{ }^{\mathrm{a}}}$ & Length $^{\mathrm{b}}(\AA)$ \\
\hline PFBA & $\mathrm{C}_{4} \mathrm{HF}_{7} \mathrm{O}_{2}$ & $\mathrm{ND}$ & 2.04 & 6.02 \\
PFPeA & $\mathrm{C}_{5} \mathrm{HF}_{9} \mathrm{O}_{2}$ & $\mathrm{ND}$ & 2.65 & 6.47 \\
PFHxA & $\mathrm{C}_{6} \mathrm{HF}_{11} \mathrm{O}_{2}$ & $\mathrm{ND}$ & 3.25 & 7.94 \\
PFHpA & $\mathrm{C}_{7} \mathrm{HF}_{13} \mathrm{O}_{2}$ & 290.8 & 3.85 & 8.96 \\
PFOA & $\mathrm{C}_{8} \mathrm{HF}_{15} \mathrm{O}_{2}$ & 179.2 & 4.46 & 9.64 \\
PFNA & $\mathrm{C}_{9} \mathrm{HF}_{17} \mathrm{O}_{2}$ & 154.3 & 5.06 & 11.77 \\
PFDA & $\mathrm{C}_{10} \mathrm{HF}_{19} \mathrm{O}_{2}$ & 50.26 & 5.66 & 12.66 \\
PFUnA & $\mathrm{C}_{11} \mathrm{HF}_{21} \mathrm{O}_{2}$ & 13.37 & 6.27 & 13.07 \\
PFDoA & $\mathrm{C}_{12} \mathrm{HF}_{23} \mathrm{O}_{2}$ & 10.89 & 6.87 & 14.08 \\
PFTrDA & $\mathrm{C}_{13} \mathrm{HF}_{25} \mathrm{O}_{2}$ & 10.14 & 7.47 & 15.10 \\
PFTeDA & $\mathrm{C}_{14} \mathrm{HF}_{27} \mathrm{O}_{2}$ & 9.920 & 8.08 & 17.15 \\
PFHxDA & $\mathrm{C}_{16} \mathrm{HF}_{31} \mathrm{O}_{2}$ & 7.280 & 9.28 & 19.87 \\
PFOcDA & $\mathrm{C}_{18} \mathrm{HF}_{35} \mathrm{O}_{2}$ & 2.960 & 10.49 & 20.98 \\
PFBS & $\mathrm{C}_{4} \mathrm{HF}_{9} \mathrm{O}_{3} \mathrm{~S}$ & $\mathrm{ND}$ & 2.72 & 7.15 \\
PFHxS & $\mathrm{C}_{6} \mathrm{HF}_{13} \mathrm{O}_{3} \mathrm{~S}$ & 67.44 & 3.93 & 10.11 \\
PFOS & $\mathrm{C}_{8} \mathrm{HF}_{17} \mathrm{O}_{3} \mathrm{~S}$ & 41.22 & 5.14 & 11.98 \\
\hline
\end{tabular}

ND: not detected.

a $\log K_{\text {ow }}$ values were provided by ChemBioDraw.

b Molecular length was cited from Zhang et al. (2013). and carbon chain length of PFCAs was showed in Fig. 2. With the length of carbon chain increasing, the inhibitory effect of PFCAs was gradually enhanced, which indicated that the carbon chain length took a leading role in their inhibitory effects. Similar trend was also observed for the three PFSAs (PFBS, PFHxS and PFOS). Among all the PFAAs, the PFOcDA, which has 18 carbon chain, displayed the strongest effect $\left(K_{\mathrm{i}}=\right.$ $2.960 \mu \mathrm{M})$, even stronger than ppGpp $\left(K_{\mathrm{i}}=3.810 \mu \mathrm{M}\right)$. In addition, PFSAs displayed significantly higher inhibitory effect than PFCAs with the same molecular length. For example, the inhibitory effect of PFOS is almost four times stronger than that of PFNA. The structural dependence of PFAA inhibitory effect is discussed in more detail later.

\subsection{LDC conformational change upon binding of PFAAs}

The binding interaction of PFAAs with LDC was characterized further by $C D$ spectroscopy. The CD spectrum of LDC itself exhibited a minimum at $225 \mathrm{~nm}$ and a maximum at $196 \mathrm{~nm}$ (Fig. 3), consistent with the presence of $\alpha$-helical and $\beta$-structure components. Using the Yang's method (Yang et al., 1986), the secondary structure of LDC was analyzed, and was found to be composed of $44 \% \alpha$-helices, $26 \% \beta$-structures, and $30 \%$ random coils, which are consistent with the crystal structure of LDC ( $41 \% \alpha$-helices, $21 \% \beta$-structures, and 38\% random coils). With addition of PFBA, PFOA, PFOS, and PFOCDA, CD spectral change was different depending on the type of the chemical. For PFBA, no appreciable change of CD spectra was detected. For PFOA, the signal had a slight decrease at $220 \mathrm{~nm}$. For PFOS, the CD intensity of LDC increased progressively and the intensity enhanced by about $21.4 \%$ when the concentration of PFOS reached $50 \mu \mathrm{M}$. Secondary structural change was 3.3\% increase in $\alpha$-helices, $2.2 \%$ increase in $\beta$-structures and 5.5\% decrease in random coils, involving approximately 9, 3 and 14 amino acid

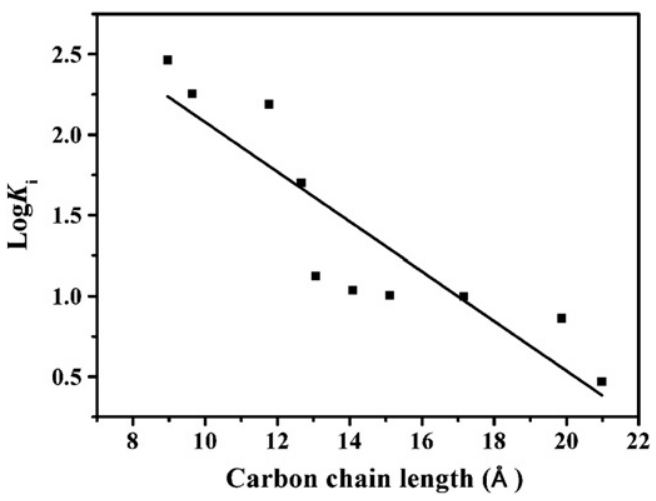

Fig. 2. The relationship between inhibition constant $\left(\log K_{\mathrm{i}}\right)$ and carbon chain length of PFAAs. 

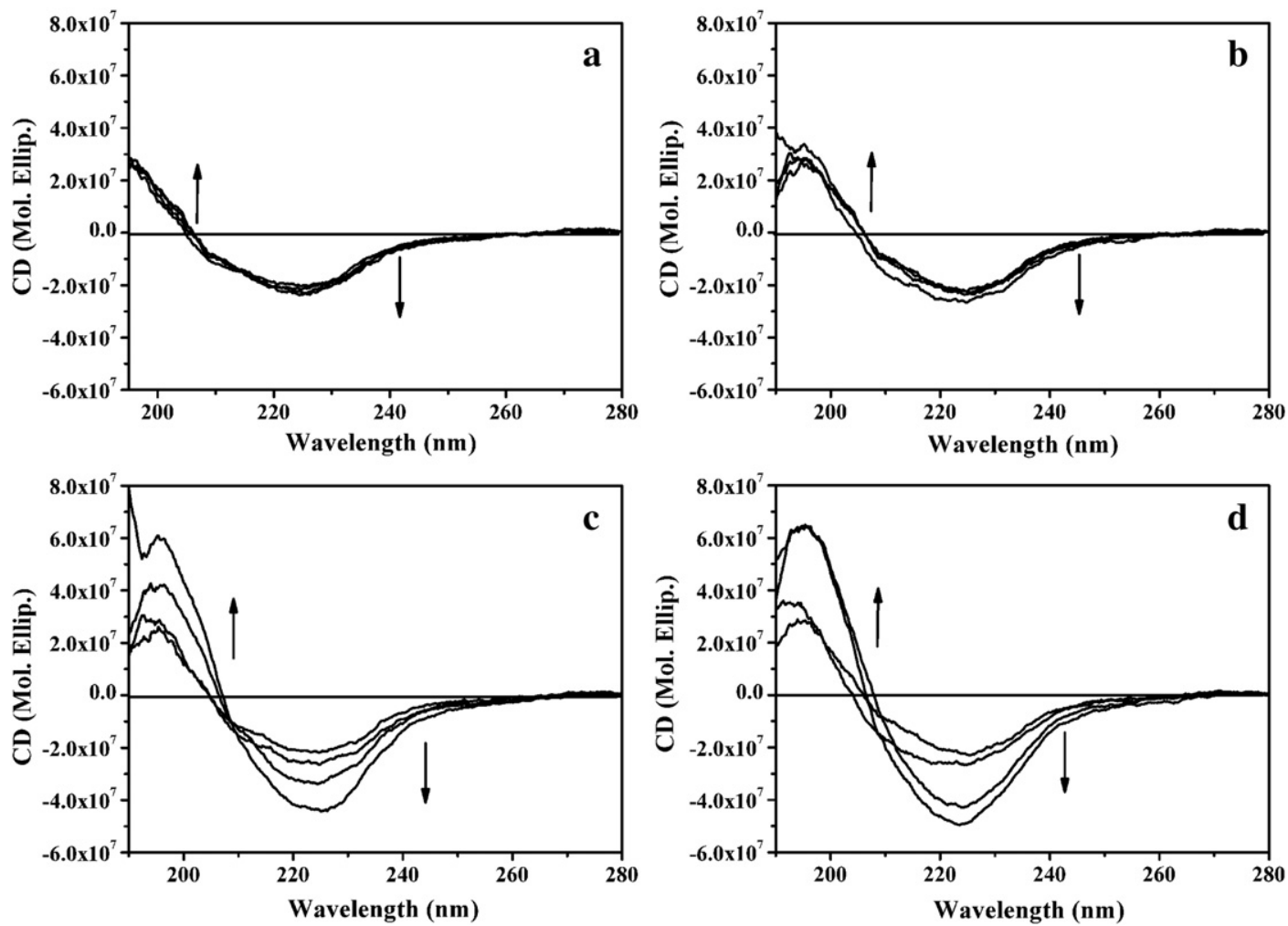

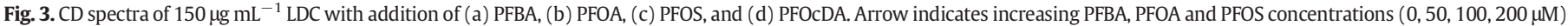
and PFOcDA concentrations $(0,10,25,50 \mu \mathrm{M})$.

residues, respectively. The most dramatic change was observed for PFOcDA, in which the intensity increased by about $114.2 \%$. Secondary structural change was found to be $8.5 \%$ increase in $\alpha$-helices, $5.4 \%$ increase in $\beta$-structures and $13.9 \%$ decrease in random coils when the concentration reached $50 \mu \mathrm{M}$, with about 25,8 and 37 residues relocated, respectively. The degree of $C D$ spectral change is in the order of PFOcDA $>$ PFOS $>$ PFOA $>$ PFBA, which is consistent with their inhibition potency in the fluorescence enzyme assay.
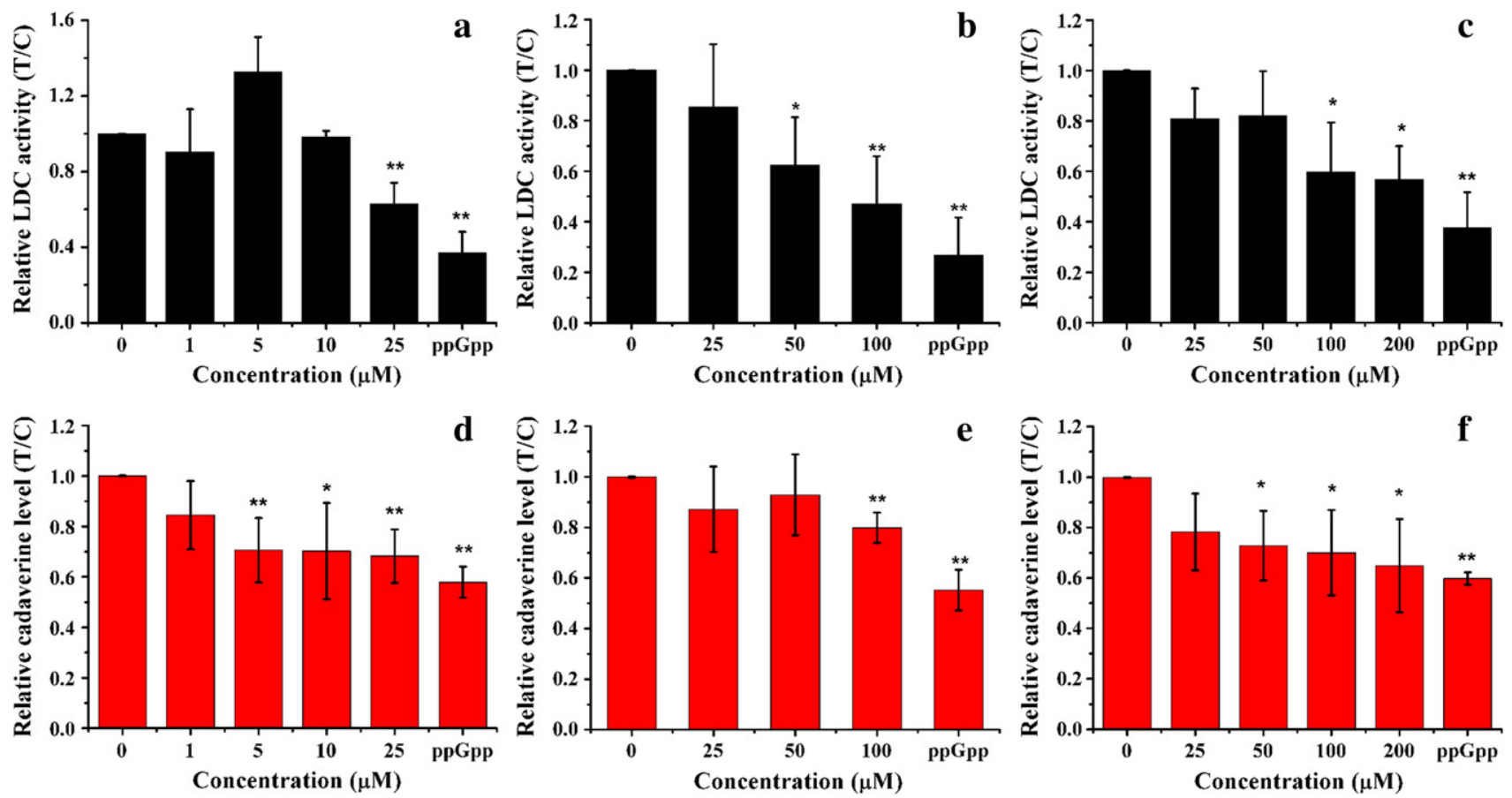

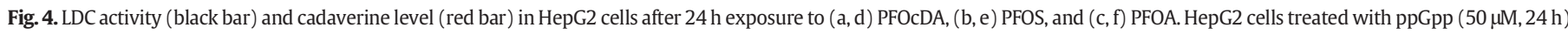

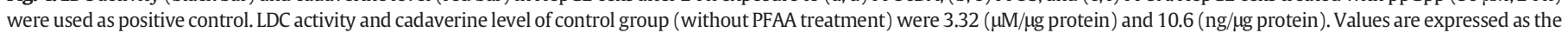

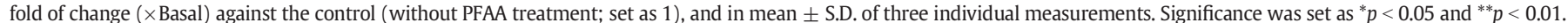




\section{a) ppGpp}

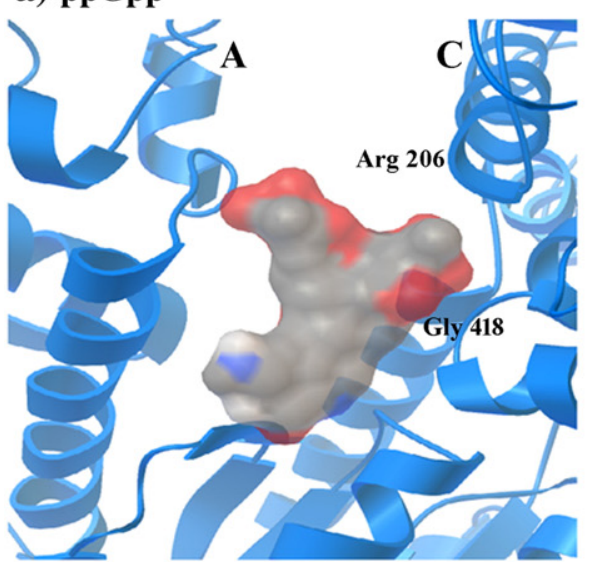

c) PFOA

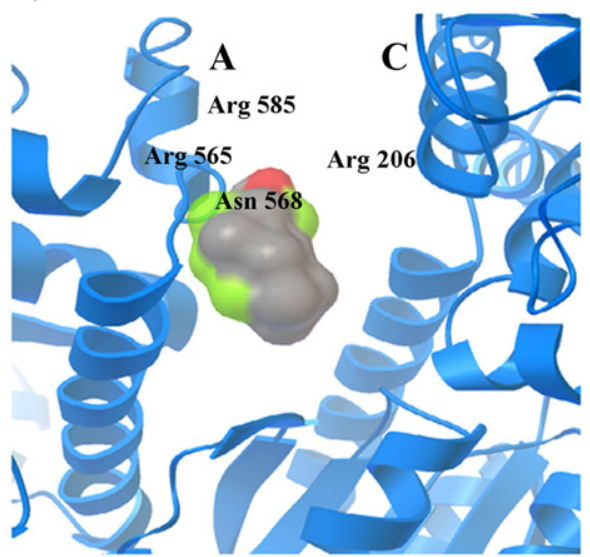

e) PFTeDA

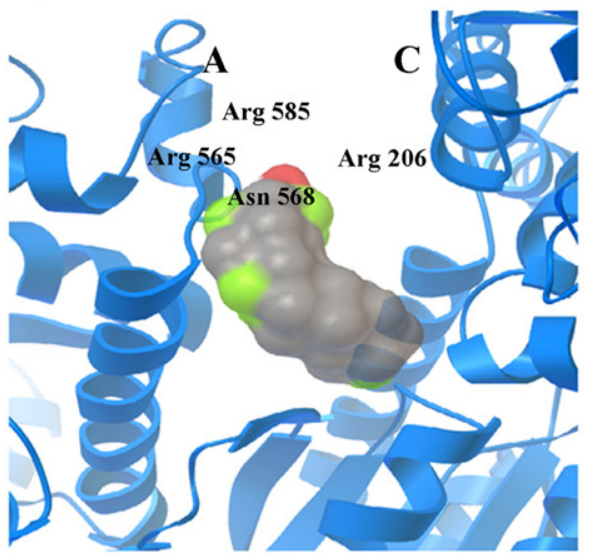

b) PFBA

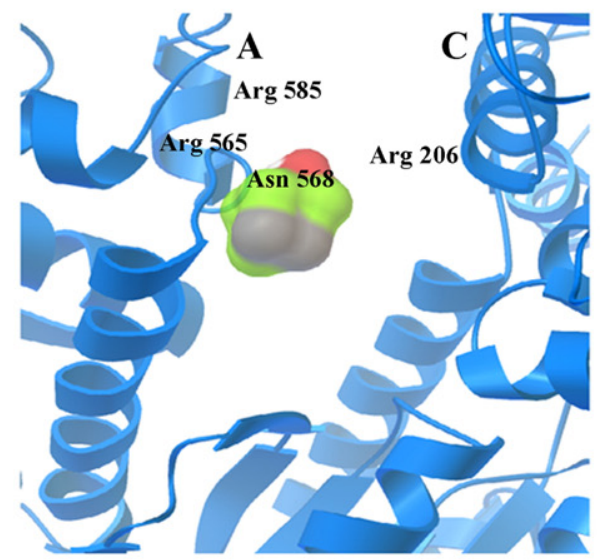

d) PFOS

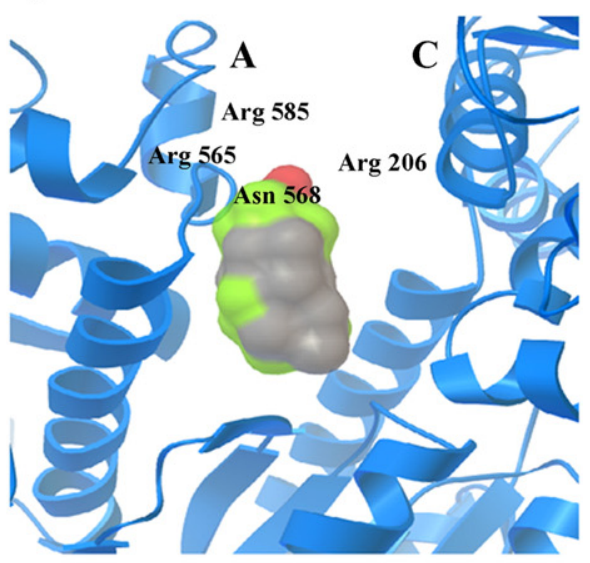

f) PFOcDA

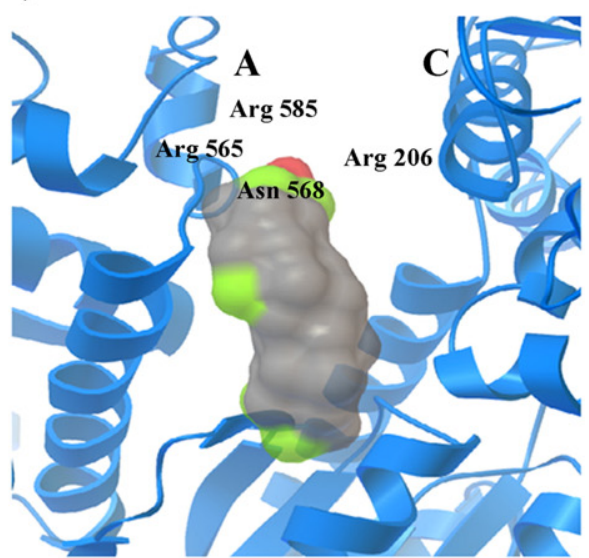

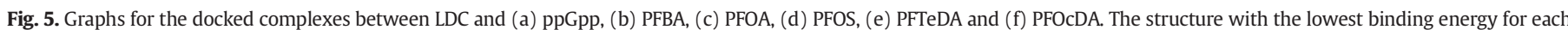
ligand is shown. PFAAs are colored by atom type (carbon in gray, oxygen in red, hydrogen in white and fluorine in yellow).

\section{4. $L D C$ activity in cells after PFAA exposure}

HepG2 cell was selected to further verify the effect of PFAAs on LDC activity in cells. It was necessary for us to first examine the effect of PFAAs on cell viability so as to obtain the dose-effect curve. MTT assay was used to detect the cell viability of HepG2 cells after $24 \mathrm{~h}$ incubation with a series of concentrations of PFOcDA $(0-50 \mu \mathrm{M})$, PFOS, PFOA and PFBA $(0-500 \mu \mathrm{M})$. Results found that the cell viability decreased in a concentration-dependent manner when exposed to PFOS, with significant viability loss at $160 \mu \mathrm{M}$. However, the toxicity of PFOA and PFBA was observed at the highest concentration $(500 \mu \mathrm{M})$ only. The cell viability did not change significantly $(<6 \%)$ after PFOcDA exposure (Fig. S6 in the Supplementary data). At non-cytotoxic concentrations, four representative PFAAs, including PFBA, PFOA, PFOS and PFOcDA, were chosen and exposed to the HepG2 cells for $24 \mathrm{~h}$. When appropriate concentration of lysine was incubated with moderate cell lysate containing $25 \mu \mathrm{g}$ protein at $37^{\circ} \mathrm{C}$ for $1 \mathrm{~h}$, cadaverine was produced and subsequently taken as a measurement of LDC enzymatic activity. The LDC enzymatic activity with or without PFAA exposure were determined. As depicted in Fig. $4 a-c$, three chemicals inhibited the LDC activity in a 
concentration-dependent manner. After normalization to the control medium (VC), LDC activity decreased from $3.32(\mu \mathrm{M} / \mu \mathrm{g}$ protein) to $1.94(\mu \mathrm{M} / \mu \mathrm{g}$ protein) upon PFOcDA exposure $(25 \mu \mathrm{M})$ and was lower by about $41.6 \%$. Similar to PFOcDA, LDC activity also had a decrease after PFOS and PFOA exposure, despite of less potency than PFOcDA. However for PFBA, the LDC activity did not change significantly (Fig. S7a in the Supplementary data). The degree of inhibitory effect is in the order of PFOcDA $>$ PFOS $>$ PFOA $>$ PFBA, which is consistent with the results obtained from the fluorescence sensing assay.

\subsection{Cadaverine level of cells under PFAA exposure}

Thereafter, we compared cadaverine concentration in the cells with or without PFAA exposure. In comparison to cells treated with control medium, PFOcDA, PFOS and PFOA treated cells had a reduced amount of cadaverine, as shown in Fig. 4d-f. Cadaverine concentration (3.35 ng/ $\mu$ g protein) decreased by $31.7 \%$ under the exposure to $25 \mu \mathrm{M}$ PFOcDA, and exhibited a similar tendency in cells exposed to PFOS and PFOA. In view of these results, we concluded that the inhibitory effect of PFAAs on the LDC activity and subsequent biological effects were evident in living cells.

\subsection{Molecular simulation of PFAAs binding with $L D C$}

In order to better understand the inhibitory effect of PFAAs, the binding interaction of PFAAs with LDC was simulated by molecular docking. The X-ray crystal structure of LDC reveals that the protein is an oligomer of five dimers that associate to form a decamer, and the dimer interface forms a narrow cleft that leads to the active site (Kanjee et al., 2011). ppGpp was first docked to LDC using AutoDock software. The results show that ppGpp resides at the interface of the cleft, and the binding pocket is made up of three contiguous parts: a hydrophobic portion that interacts with the guanosine base of ppGpp, and two other hydrophobic portions that interact with 3'- and 5'-phosphate respectively. Three hydrogen bonds are formed between the phosphate group and the side chain of Arg206 and the backbone amide of Gly418 (Fig. 5a). According to the crystallographic structure of LDC/ppGpp (Kanjee et al., 2011), the ppGpp molecule is located at the dimer interface and its interaction with LDC involves guanosine base, $3^{\prime}$ - and 5'-phosphate of ppGpp. Besides, the 3'-phosphate forms hydrogen bonds to both the side chain of Arg206 and the backbone amide of Gly418 of LDC. In comparison, our modeling results are in good agreement with those reported in the literature.

Using the same docking parameters, 16 PFAAs were docked with LDC. All the PFAAs were found to bind to LDC at the same cleft as ppGpp (Figs. 5b-f and S8 in the Supplementary data). The charged head group of a PFAA interacts with residues Arg565, Asn568, Arg585 and Arg206 by hydrogen bonds, and the hydrophobic tail makes contact with the non-polar amino acid residues in the cleft. Obviously, the contact area of long chain PFAAs with the non-polar portion of LDC is larger than that of medium chain (7-10 carbons) PFAAs. However for short chain PFAAs, the binding mode was similar among them but significantly different from the long chain PFAAs. The carbon chain was so short that it could not fit into the binding cleft. This may explain why their binding affinity with the enzyme increases with carbon number and PFOcDA possesses the strongest binding affinity. Moreover, according to the docking results, the binding energy between PFAAs and LDC was calculated. The order of binding energy is long chain $(-3.98$ to $-3.51 \mathrm{kcal} / \mathrm{M})<$ medium chain $(-2.53$ to $-2.46 \mathrm{kcal} / \mathrm{M})<$ short chain $(-1.62$ to $-1.41 \mathrm{kcal} / \mathrm{M})$ PFAAs, with PFOcDA substantially lower than all other PFAAs. As a matter of fact, there exists a good correlation between the inhibition constant and $\log K_{\text {ow }}$ of PFCAs (correlation coefficient $R^{2}=0.87$ ) (Fig. S9 in the Supplementary data), illustrating the important role of hydrophobic forces in PFAA/LDC interaction. However, since possibly electrostatic interaction is also involved, the nature of the PFAA head group should make a difference. Sulfonate carries more negative charges, and is also capable of forming stronger electrostatic interactions with LDC than carboxylate. This may explain why some PFSAs interact stronger with LDC than PFCAs even if their molecular length, $\log K_{\mathrm{ow}}$ and hydrogen bonding are similar.

\section{Conclusions}

Inhibitory effect of 16 PFAAs on LDC activity was investigated by a fluorescence enzyme assay, CD spectroscopy, cell assay and molecular docking analysis. The inhibition constants of PFAAs were obtained for the first time with PFOcDA possessing the strongest inhibitory effect. The inhibitory effect was found to be highly dependent on the hydrophobicity and functional group of PFAAs. The structural dependence was rationalized by structure-activity relationship analysis that took into account the dominant hydrophobic forces and hydrogen bonding. Moreover, the results at cellular level further confirmed that the LDC activity was inhibited remarkably by PFAAs, leading to decreased cadaverine level in living cells. The combined results suggest that LDC might be a potential biological target for PFAA toxic effects.

\section{Acknowledgments}

This work was supported by the National Basic Research Program of China (2011CB936001, 2010CB933502), National Natural Science Foundation of China $(21077124,21375143,21377142,21277158$, 21177138), and Chinese Academy of Sciences (YSW2013A01).

\section{Appendix A. Supplementary data}

Supplementary data to this article can be found online at http://dx. doi.org/10.1016/j.scitotenv.2014.07.034.

\section{References}

Andersen ME, Butenhoff JL, Chang SC, Farrar DG, Kennedy Jr GL, Lau C, et al. Perfluoroalkyl acids and related chemistries-toxicokinetics and modes of action. Toxicol Sci 2008; 102:3-14.

Bagni N, Tassoni A. Biosynthesis, oxidation and conjugation of aliphatic polyamines in higher plants. Amino Acids 2001;20:301-17.

Benninghoff AD, Bisson WH, Koch DC, Ehresman DJ, Kolluri SK, William DE. Estrogen-like activity of perfluoroalkyl acids in vivo and interaction with human and rainbow trout estrogen receptors in vitro. Toxicol Sci 2011;120:42-58.

Calandra RS, Rulli SB, Frungieri MB, Suescun MO, Gonzalez-Calvar SI. Polyamines in the male reproductive system. Acta Physiol Pharmacol Ther Latinoam 1996;46:209-22.

Chang SC, Thibodeaux JR, Eastvold ML, Ehresman DJ, Bjork JA, Froehlich JW, et al. Negative bias from analog methods used in the analysis of free thyroxine in rat serum containing perfluorooctanesulfonate (PFOS). Toxicology 2007;234:21-33.

Chen YM, Guo L-H. Fluorescence study on site-specific binding of perfluoroalkyl acids to human serum albumin. Arch Toxicol 2009;83:255-61.

Conder JM, Hoke RA, De Wolf W, Russell MH, Buck RC. Are PFCAs bioaccumulative? A critical review and comparison with regulatory lipophilic compounds. Environ Sci Technol 2008;42:995-1003.

Copeland RA. Mechanistic considerations in high-throughput screening. Anal Biochem 2003;320:1-12.

DeWitt JC, Shnyra A, Badr MZ, Loveless SE, Hoban D, Frame SR, et al. Immunotoxicity of perfluorooctanoic acid and perfluorooctane sulfonate and the role of peroxisome proliferator-activated receptor alpha. Crit Rev Toxicol 2009;39:76-94.

Flores HE, Galston AW. Analysis of polyamines in higher plants by high performance liquid chromatography. Plant Physiol 1982;69:701-6.

Gao Y, Li XX, Guo L-H. Assessment of estrogenic activity of perfluoroalkyl acids based on ligand-induced conformation state of human estrogen receptor. Environ Sci Technol 2013;47:634-41.

Gerner EW, Meyskens FL. Polyamines and cancer: old molecules, new understanding. Nat Rev Cancer 2004;4:781-92.

Giesy JP, Kannan K. Perfluorochemical surfactants in the environment. Environ Sci Technol 2002;36:146A-52A.

Guyton KZ, Chiu WA, Bateson TF, Jinot J, Siegel Scott C, Brown RC, et al. A reexamination of the PPAR- $\alpha$ activation mode of action as a basis for assessing human cancer risks of environmental contaminants. Environ Health Perspect 2009;117:1664-72.

Hebert PC, MacManus-Spencer LA. Development of a fluorescence model for the binding of medium- to long-chain perfluoroalkyl acids to human serum albumin through a mechanistic evaluation of spectroscopic evidence. Anal Chem 2010;82:6463-71.

Henning A, Bakirci H, Nau WM. Label-free continuous enzyme assays with macrocyclefluorescent dye complexes. Nat Methods 2007;4:629-32.

Houde M, Martin JW, Letcher RJ, Solomon KR, Muir DCG. Biological monitoring of polyfluoroalkyl substances: a review. Environ Sci Technol 2006;40:3463-73. 
Ito Y, Yamanoshita O, Asaeda N, Tagawa Y, Lee CH, Aoyama T, et al. Di(2-ethylhexyl) phthalate induces hepatic tumorigenesis through a peroxisome proliferatoractivated receptor alpha-independent pathway. J Occup Health 2007;49:172-82.

Kanjee U, Gutsche I, Alexopoulos E, Zhao BY, EI Bakkouri M, Houry WA. Linkage between the bacterial acid stress and stringent responses: the structure of the inducible lysine decarboxylase. EMBO J 2011;30:931-44.

Lau C, Thibodeaux JR, Hanson RG, Rogers JM, Grey BE, Stanton ME, et al. Exposure to perfluorooctane sulfonate during pregnancy in rat and mouse. II: postnatal evaluation. Toxicol Sci 2003;74:382-92.

Lau C, Anitole K, Hodes C, Lai D, Hutchens AP, Seed J. Perfluoroalkyl acids: a review of monitoring and toxicological findings. Toxicol Sci 2007;99:366-94.

Leistner E, Spenser ID. Biosynthesis of the piperidine nucleus. Incorporation of chirally labeled (1-3H) cadaverine. J Am Chem Soc 1973;95:4715-25.

Liu XQ, He YM, Ren YS, Cai FQ Wang YL. Determining the content of polyamine in cell lysis liquid by high performance liquid chromatography. Chin Hosp Pharm J 2010; 30:1159-61.

Michael JP. Indolizidine and quinolizidine alkaloids. Nat Prod Rep 2008;25:139-65.

Morris GM, Goodsell DS, Halliday RS, Huey R, Hart WE, Belew RK, et al. Automated docking using a Lamarckian genetic algorithm and an empirical binding free energy function. J Comput Chem 1998;19:1639-62.

Morris GM, Huey R, Lindstrom W, Sanner MF, Belew RK, Goodsell DS, et al. AutoDock4 and AutoDockTools4: automated docking with selective receptor flexibility. J Comput Chem 2009;30:2785-91.

Nau WM, Ghale G, Hennig A, Bakirci H, Bailey DM. Substrate-selective supramolecular tandem assays: monitoring enzyme inhibition of arginase and diamine oxidase by fluorescent dye displacement from calixarene and cucurbituril macrocycles. J Am Chem Soc 2009;131:11558-70.
Ohmiya S, Saito K, Murakoshi IIn: Cordell GA, editor. Lupine alkaloids. The alkaloids, Chemistry and PharmacologyNew York: Academic Press; 1995. p. 1-114.

Phan APH, Ngo TT, Lenhoff HM. Spectrophotometric assay for lysine decarboxylase. Anal Biochem 1982;120:193-7.

Raymer JH, Michael LC, Studabaker WB, Olsen GW, Sloan CS, Wilcosky T, et al. Concentrations of perfluorooctane sulfonate (PFOS) and perfluorooctanoate (PFOA) and their associations with human semen quality measurements. Reprod Toxicol 2012;33: 419-27.

Sundström M, Ehresman DJ, Bignert A, Butenhoff JL, Olsen GW, Chang SC, et al. A temporal trend study (1972-2008) of perfluorooctanesulfonate, perfluorohexanesulfonate, and perfluorooctanoate in pooled human milk samples from Stockholm, Sweden. Environ Int 2011;37:178-83.

Thorsten S, Daniela M, Brunn H. Toxicology of perfluorinated compounds. Environ Sci Eur 2011;23:1-52.

Wang F, Liu W, Jin Y, Dai J, Zhao H, Xie Q, et al. Interaction of PFOS and BDE-47 coexposure on thyroid hormone levels and $\mathrm{TH}$-related gene and protein expression in developing rat brains. Toxicol Sci 2011;121:279-91.

Weiss JM, Andersson PL, Lamoree MH, Lenonards PEG, van Leeuwen SPJ, Hamers T. Competitive binding of poly- and perfluorinated compounds to the thyroid hormone transport protein transthyretin. Toxicol Sci 2009;109:206-16.

White SS, Fenton SE, Hines EP. Endocrine disrupting properties of perfluorooctanoic acid. J Steroid Biochem Mol Biol 2011:127:16-26.

Yang JT, Wu CSC, Martinez HM. Calculation of protein conformation from circulardichroism. Methods Enzymol 1986;130:208-69.

Zhang LY, Ren X-M, Guo L-H. Structure-based investigation on the interaction of perfluorinated compounds with human liver fatty acid binding protein. Environ Sci Technol 2013;47:11293-301. 